\title{
P I6-37. HIV controllers with weak CD8+ $T$ cell responses maintain a tight control of infection despite carrying infectious viruses
} A Saez-Cirion*1, M Sinet ${ }^{2}$, S Shin ${ }^{1}$, A Urrutia ${ }^{2}$, P Versmisse ${ }^{1}$, F Boufassa ${ }^{3}$, C Rouzioux ${ }^{4}$, O Lambotte ${ }^{2}$, A Venet ${ }^{2}$ and G Pancino ${ }^{1}$

Address: ${ }^{1}$ Régulation des Infections Rétrovirales, Institut Pasteur, Paris, France, ${ }^{2}$ Inserm U802, Le Kremlin-Bicêtre, France, ${ }^{3}$ Inserm U822, Le Kremlin-Bicêtre, France and ${ }^{4} \mathrm{AP}-\mathrm{HP}, \mathrm{CHU}$ Necker-Enfants Malades, Laboratoire de Virologie, Paris, France

* Corresponding author

from AIDS Vaccine 2009

Paris, France. 19-22 October 2009

Published: 22 October 2009

Retrovirology 2009, 6(Suppl 3):P266 doi:10.1 186/1742-4690-6-S3-P266

This abstract is available from: http://www.retrovirology.com/content/6/S3/P266

(C) 2009 Saez-Cirion et al; licensee BioMed Central Ltd.

\section{Background}

HIV controllers (HIC) are rare HIV-1-infected individuals who durably maintain undetectable viremia in the absence of therapy. Viral control in HIC is usually associated to strong functional HIV-specific CD8+ T cell responses. Accordingly, we have shown that $\mathrm{CD} 8+\mathrm{T}$ cells from most HIC have a strong capacity ex vivo to suppress HIV-1 infection. We have further characterized the HIVsuppressive capacity of CD8+ T cells and the mechanisms of HIV control in 19 HICs.

\section{Methods}

HIV-specific CD8+ T cells were quantified by IFNg ELISPOT and characterized (activation, maturation and proliferation) by flow cytometry. Their capacity to suppress HIV infection was evaluated by p24 ELISA of supernatants of co-cultures with autologous CD4+ T cells. Replication of infecting viruses was assessed upon PHA+IL2 stimulation of CD4+ T cells. Infectious capacity and tropism of HIC viruses were evaluated in allogenic CD4+ $\mathrm{T}$ cells and CCR5+ or CXCR4+ cells lines.

\section{Results}

The capacity of CD8+ T cells from 14/19 HIC to suppress HIV infection was strong and associated to the frequency of Gag-specific CD8+ T cell responses (strong responder, SR, HIC). However 5/19 HIC have low numbers of HIVspecific CD8+ T cells with very low activation levels (weak responder, WR, HIC). WR HIC seems to control viremia more tightly than SR HIC, who may require some viral replication to boost the HIV-specific response. Moreover, WR HIC maintains their levels of CD4+ T cells while some lost is often observed in SR HIC. Infecting viruses from WR HIC are readily detected upon activation of CD4+ T cells. Viruses from at least 3 WR HIC are infectious and X4 or dualtropic.

\section{Conclusion}

Although our results underline the importance of Gagspecific CD8+ T cell responses in HIV control, the identification of WR suggest the existence of additional mechanisms of control of infection in HIC. 